\title{
Where is the Lead? Inappropriate Implantable Cardioverter- Defibrillator Shock Caused by Extreme Twiddling
}

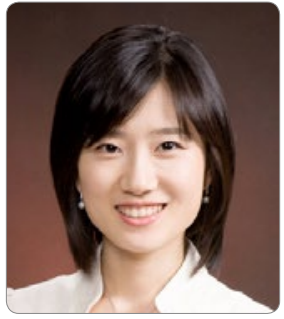

So-Ryoung Lee

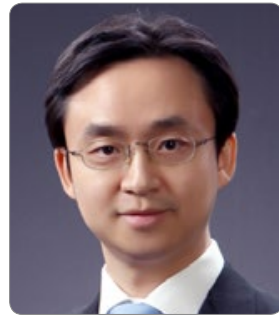

Eue-Keun Choi
So-Ryoung Lee, MD; Eue-Keun Choi, MD, PhD

Division of Cardiology, Department of Internal Medicine, Seoul National University Hospital, Seoul, Republic of Korea
Received: November 2, 2016

Revision Received: December 13, 2016

Accepted: December 14, 2016

Correspondence: Eue-Keun Choi, MD, PhD

Department of Internal Medicine, Seoul National

University Hospital 101, Daehak-ro, Jongno-gu,

Seoul 03080, Republic of Korea

Tel: +82-2-2072-0688 Fax: +82-2-762-9662

E-mail: choiek17@snu.ac.kr

Copyright (C) 2016 The Official Journal of Korean Heart Rhythm Society Editorial Board \& MMK Co., Ltd.

\begin{abstract}
A 43-year-old man who had received mitral and aortic valve replacement surgery underwent the implantation of an implantable cardioverter defibrillator (ICD) for sustained ventricular tachycardia. The patient presented with a sudden jolting sensation in his left upper chest area one year after the device implantation. He had a history of vigorous upper body exercise during the several months of the follow-up period. Device interrogation revealed complete sensing and capture failure. The ventricular lead impedance was in the normal range, but the high voltage impedance had dropped to less than $10 \Omega$. Four inappropriate shocks for ventricular fibrillation had been delivered due to over-sensing of the atrial signal on the ventricular lead. Chest radiography showed ventricular lead displacement with extreme rotation and flipping-over of the generator. In the lead revision operation, the old ventricular lead was extracted and replaced, and the generator was fixed more deeply in the pocket with a non-absorbable ligature.
\end{abstract}

Key Words: - Implantable Cardioverter-Defibrillator -Twiddler's Syndrome - Inappropriate Shock

\section{Introduction}

Twiddler's syndrome is a pacemaker or implantable cardioverter defibrillator (ICD) malfunction caused by manipulation of the device and subsequent displacement of the leads. ${ }^{1}$ While it has been well described among pacemaker patients, it is relatively uncommon in patients with an ICD. ${ }^{2-7}$ We report the case of a patient with inappropriate ICD shocks caused by ventricular lead displacement from the extreme rotation of the ICD generator following vigorous upper extremity exercise.

\section{Case}

A 43-year-old man $(108 \mathrm{~kg}, 194 \mathrm{~cm})$ presented to the emergency department with dizziness and palpitations that were diagnosed as sustained ventricular tachycardia (VT). 
Monomorphic VT (a QRS duration of $174 \mathrm{~ms}$ ) with a $200 \mathrm{bpm}$ rate was observed. The QRS morphology showed a right bundle branch block-like pattern (monophasic $\mathrm{R}$ wave in lead $\mathrm{V}_{1}$ ) with late precordial transition at $\mathrm{V}_{4-5}$, a northwest axis $\left(240^{\circ}\right)$, positivity in the aVR, isoelectricity in the aVL, and QS in lead I. Based on the electrocardiographic characteristics, the site of origin of the VT was suspected to be the left ventricular (LV) mid-inferolateral region. Echocardiography showed LV dilatation and dysfunction (LV end-diastolic dimension: $66 \mathrm{~mm}, \mathrm{LV}$ ejection fraction: 25\%). The patient had undergone mitral and aortic valve replacement surgery for severe mitral and aortic valve regurgitation after rheumatic fever approximately 30 years prior. Before the sustained VT event, he had been prescribed nebivolol $2.5 \mathrm{mg}$ once-daily for hypertension and mild LV dysfunction (LV ejection fraction $42 \%)$, and warfarin for mechanical valves. The patient showed normal coronary arteries on the coronary angiography and did not present any transient or correctable causes of VT, including electrolyte imbalance.

Considering that the spontaneous VT was related to valvular cardiomyopathy and that the patient had both prosthetic aortic and mitral valves, we decided to implant an ICD rather than perform a catheter ablation for VT. A single-chamber dual-coil cardioverter defibrillator (Ellipse VR MRI, St Jude Medical Inc., Saint Paul, MN, USA) was implanted. A ventricular active fixation lead (Durata 7120Q, St Jude Medical Inc.) was introduced through the left axillary vein and screwed to the right ventricular apex (Figure 1A). The generator was implanted under the skin without anchoring. Radiography performed immediately after implantation showed that both the lead and generator were well positioned (Figure 1B and 1C). Device interrogation showed good function (sensing threshold $11.8 \mathrm{mV}$, capture threshold 0.75 V, V impedance $630 \Omega$, High Voltage $[\mathrm{HV}]$ impedance $43 \Omega$ ).
A

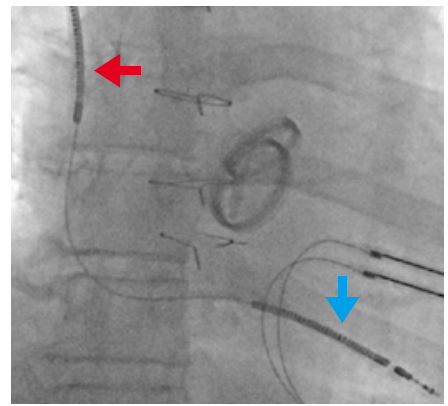

A. Fluoroscopy image during ICD implantation.

D

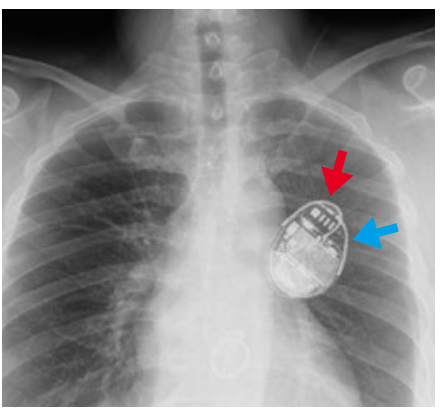

D. Chest radiogram (posteroanterior view) shows displacement of the ventricular lead around the generator.
B

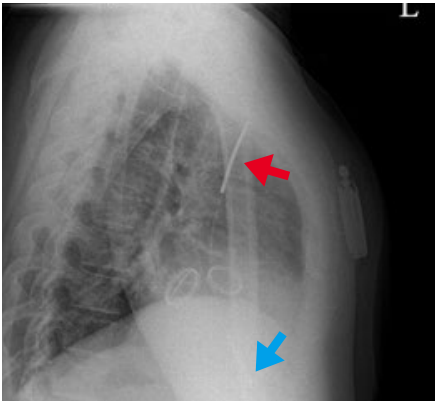

C

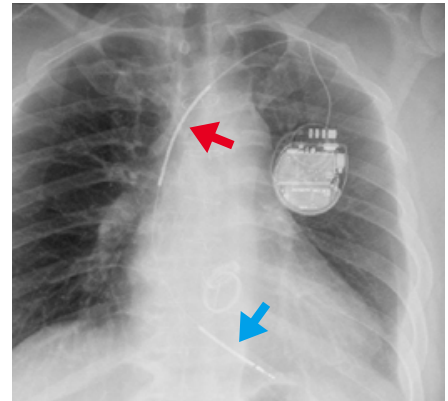

$B$ and $C$. Chest radiograms obtained immediately after ICD implantation. Posteroanterior $(C)$ and lateral (B) view.

E

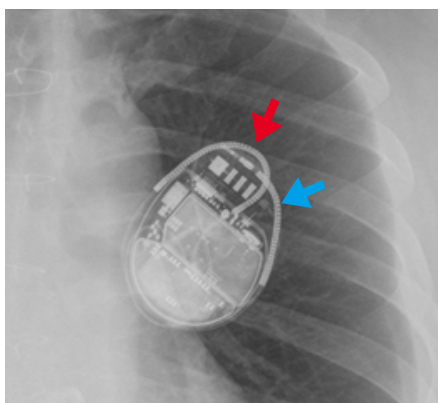

F

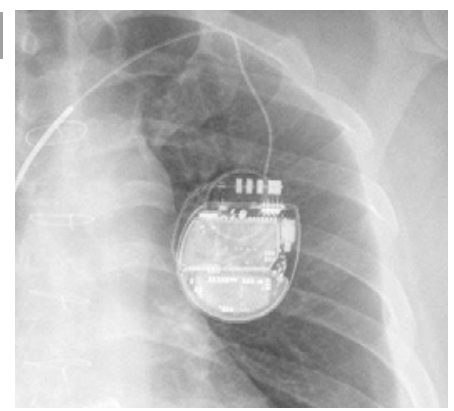

$E$ and $F$. Magnified image focused on the generator, after lead displacement (E) and immediately after ICD implantation (F). Compared with panel $F$, there has been a reversal from left to right in panel $E$, which suggests extreme rotation and flipping-over of the generator. Red arrow indicates SVC coil and blue arrow indicates RV coil.

Figure 1. Chest radiograms show the positions of the dual-coil active ICD lead and generator. ICD, implantable cardioverter-defibrillator; RV, right ventricle; SVC, superior vena cava. 
Throughout the regular follow-up period, several short, nonsustained VT events were recorded under amiodarone medication. Five months after the ICD implantation, the patient was lost to follow-up for 7 months. In his last outpatient clinic visit, the ICD interrogation had shown normal function (sensing threshold $8.0 \mathrm{mV}$, capture threshold $1.0 \mathrm{~V}, \mathrm{~V}$ impedance $390 \Omega$, HV impedance $42 \Omega$ ).

The patient revisited the outpatient clinic with a sudden jolting sensation in the left upper chest area. ICD interrogation revealed sensing and capture failure of the ventricular lead. Although the impedance of the ventricular lead had increased slightly from 390 $\Omega$ to $800 \Omega$, the HV impedance had fallen to less than $10 \Omega$ (normal range; 10-100 $\Omega$ ). Seven episodes of ventricular fibrillation (VF) and 4 deliveries of shocks for VF were recorded. The ventricular electrogram revealed over-sensing of the atrial signal ( $p$-wave) on the ventricular lead that had been misdiagnosed as VF, causing inappropriate shock delivery (Figure 2). A chest $X$-ray showed ventricular lead displacement with extreme rotation and flipping-over of the generator (Figure $1 \mathrm{E}$ and $1 F)$.

Since the last outpatient clinic visit, the patient had been performing vigorous upper extremity exercise to lose weight, including weightlifting, stretching, and swinging his arms around. The patient denied manipulation of the device pocket. We suspected that the device had rotated spontaneously and flipped over during the vigorous exercise. Simultaneously with the device movement, the ventricular lead had been drawn from the right ventricular apex and had curled around the generator in the pocket. In a second operation, the old ventricular lead was extracted and a new active fixation ventricular lead (Durata 7120 Q, dual coil screw lead, St. Jude Medical Inc.) was implanted in the right ventricular mid-septum area. The generator was fixed more deeply in the pocket using a non-absorbable ligature.

\section{Discussion}

Twiddler's syndrome is relatively rare in patients with ICD. This may be because ICD generators are usually bigger, have less capacious pockets, and are anchored by non-absorbable ligatures more frequently than are pacemakers. ${ }^{2-8}$ Most cases are attributable to the deliberate or subconscious manipulation of the generator by patients.

In this case, the patient was obese and the abundant fat tissue around the generator pocket might have facilitated the drooping of the generator. Although the slack of the ventricular lead had
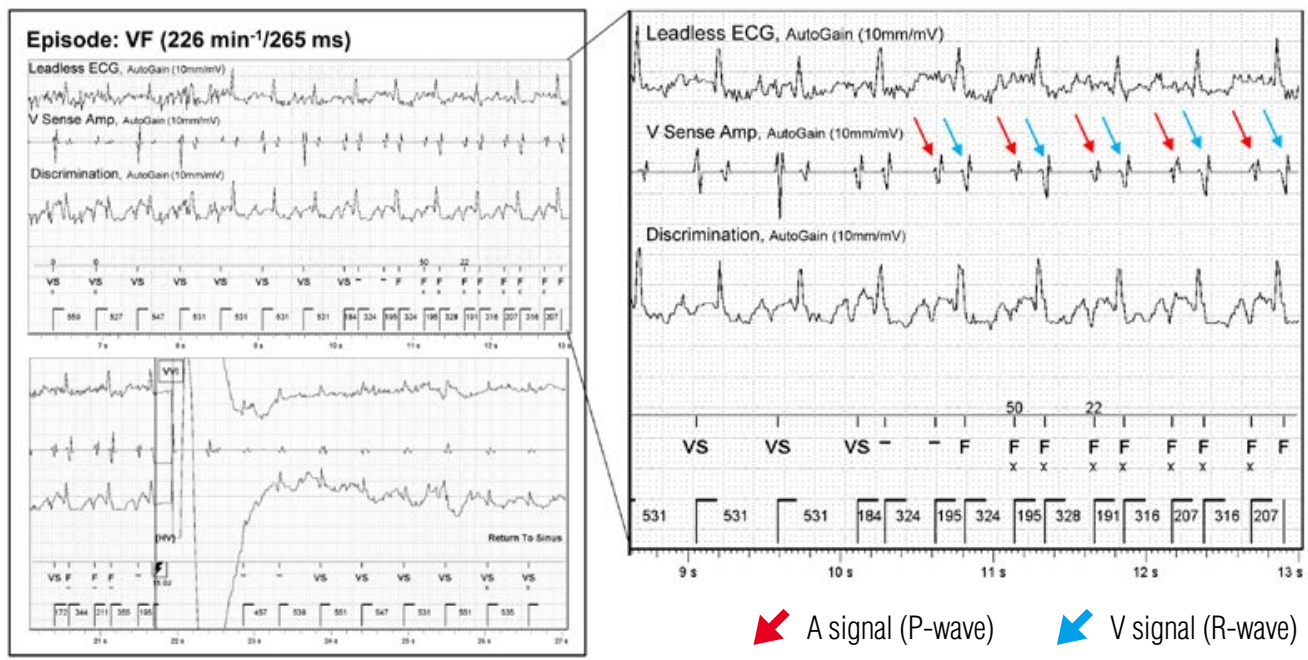

Figure 2. Stored ventricular electrogram of the ventricular fibrillation (VF) episode. Over-sensing of the atrial signal caused a VF misdiagnosis that led to inappropriate shock delivery. The red arrow indicates the atrial signal (p-wave) and the blue arrow indicates the ventricular signal (r-wave).

$E C G$, electrocardiogram; $V F$, ventricular fibrillation. 
been considered sufficient at implantation, it had changed in the upright position. Considering the patient's tall stature, the length of the redundant lead had been just enough to make one coil. According to previous reports, obesity, advancing age, the female sex, and a pendant breast have been suggested as risk factors of Twiddler's syndrome, as patients with these characteristics are at higher risk of having the generator rotate in its pocket on account of loose subcutaneous tissue. ${ }^{910}$ In addition, although the patient denied deliberate spinning of the generator, he did perform vigorous upper body exercise despite warnings. Hence, the inadequate anchoring of the generator and movement of the same side arm caused complete retraction of the affected lead, with subsequent encircling around the generator. Therefore, a short circuit between the HV coil and the generator had occurred, resulting in a HV impedance drop. Considering the normal range of ventricular lead impedance, a lead defect such as an insulation break was not strongly suspected. Minimization of the pocket size and suturing of the device deep into the pectoral muscle fascia might help to prevent this problem.

\section{References}

1) Bayliss CE, Beanlands DS, Baird RJ. The pacemaker-twiddler's syndrome: a new complication of implantable transvenous pacemakers. Can Med Assoc J. 1968;99:371-373.
2) Mehta D, Lipsius M, Suri RS, Krol RB, Saksena S. Twiddler's syndrome with the implantable cardioverter-defibrillator. Am Heart J. 1992;123:1079-1082.

3) Avitall B, Stormo A, Barragry T, Axtel K, Hare J. Sudden cardiac death: twiddler's syndrome with an implantable cardioverter defibrillator. Am Heart J. 1994;128:833-836.

4) Boyle NG, Anselme F, Monahan KM, Beswick P, Schuger CD, Zebede J, Josephson ME. Twiddler's syndrome variants in ICD patients. Pacing Clin Electrophysiol. 1998;21:2685-2687.

5) Chemello D, Subramanian A, Cameron D. Twiddler syndrome with 180degrees rotation of an implantable cardioverter defibrillator generator resulting in malfunction of one of the shocking coils. Europace. 2009;11:1259.

6) Ahmed FZ, Allen S, Zaidi A. Extreme Twiddling: Unusual Presentation of an Old Foe. J Clin Exp Cardiolog. 2012:3;219-220.

7) Carweg C, Alzand BS, Willems R. Twiddler syndrome causing an inappropriate implantable cardioverter-defibrillator shock. Eur Heart J. 2014;35:516.

8) Nicholson WJ, Tuohy KA, Tikemeier P. Twiddler's syndrome. $N$ Engl J Med. 2003;348:1726-1727.

9) Khaliullah M, Khanna SK, Gupta U, Padmavati S. Pacemaker twiddler's syndrome: a note on its mechanism. J Cardiovasc Surg (Torino). 1979;20:95-100.

10) Pereira PL, Trübenbach J, Farnsworth CT, Huppert PE, Claussen CD. Pacemaker and defibrillator twiddler's syndrome. Eur $J$ Radiol. 1999;30:67-69. 